\title{
Young stellar populations in several photometric systems
}

\author{
Léo Girardi \\ Osservatorio Astronomico di Trieste, \\ Via G.B. Tiepolo 11, I-34131 Trieste, Italia \\ Gianpaolo Bertelli \\ Consiglio Nazionale delle Richerche, Dipartimento di Astronomia, \\ Università di Padova, Vicolo dell'Osservatorio 5, I-35122 Padova, Italia \\ Cesare Chiosi and Paola Marigo \\ Dipartimento di Astronomia, Università di Padova, \\ Vicolo dell'Osservatorio 5, I-35122 Padova, Italia
}

\begin{abstract}
We report on two recent improvements to the library of stellar models from Padova. ( $i$ ) A new set of massive stellar models has been computed for the metallicity $Z=0.001$, thus filling an important gap in the previously existing library. ( $i i$ ) We started a project aiming to convert the complete set of isochrones into a wide variety of photometric systems.
\end{abstract}

The Padova library of stellar models is widely used in the modeling of resolved young (and old) stellar populations in Local Group galaxies. We report on two recent improvements to this database.

A new set of models for massive stars has been computed for the metallicity $Z=0.001$ (Figure 1). They fill the previous gap in the extended Padova grids (Bertelli et al. 1994, and references therein), which already included massive stars for metallicities $Z=0,0.0001,0.0004,0.004,0.008,0.019$, and 0.05 , as well as the corresponding low- and intermediate-mass stars (Bertelli et al. 1994; Girardi et al. 2000; Marigo et al. 2001). The $Z=0.001$ tracks were computed with convective core overshooting, OPAL-opacities, and mass-loss rates according to de Jager et al. (1988) and Kudritzki et al. (1989). Tracks and isochrones are available at http://pleiadi.pd.astro.it.

The complete sets of isochrones are being converted into a wide variety of photometric systems ( $c f$. Girardi et al. 2002). Although the presently-used spectral library covers a large region of the $\log g v s . \log T_{\text {eff }}$ plane, it presents some limitations, especially affecting massive stars. Nonetheless, these models allow us to have a first assessment of the expected location of massive stars in still poorly-known photometric systems, for which huge photometric databases are already being collected and released. As examples, we mention the ESO/MPIWFI camera (Figure 2), the ultraviolet broad-band filters of HST-WFPC2, and the recently-defined sloan Digital sky Survey systems. In addition, our formal- 

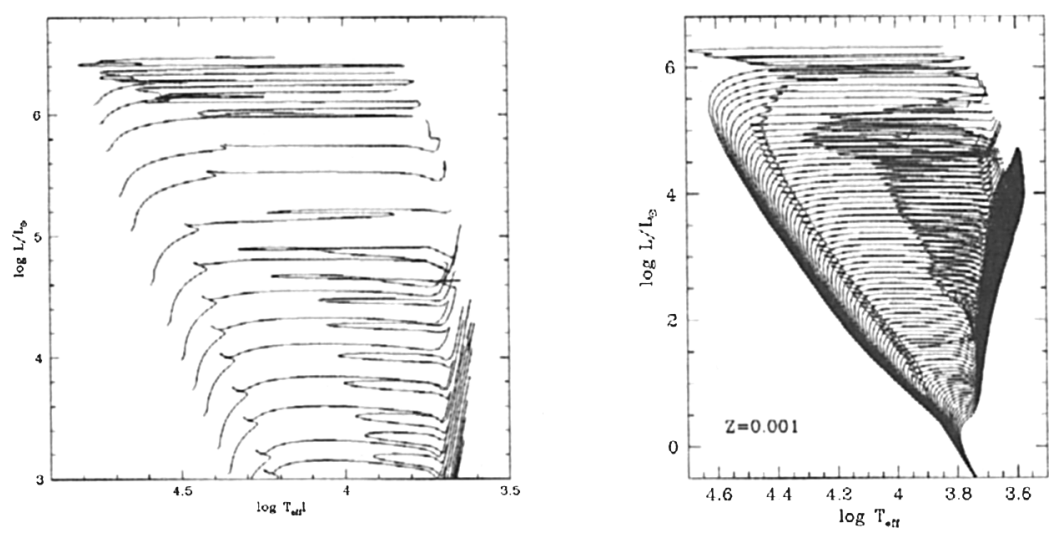

Figure 1. New set of evolutionary tracks for $[Z=0.001, Y=0.230]$, for initial masses between 7 and $100 \mathrm{M}_{\odot}$ (left), and the related isochrones (right).
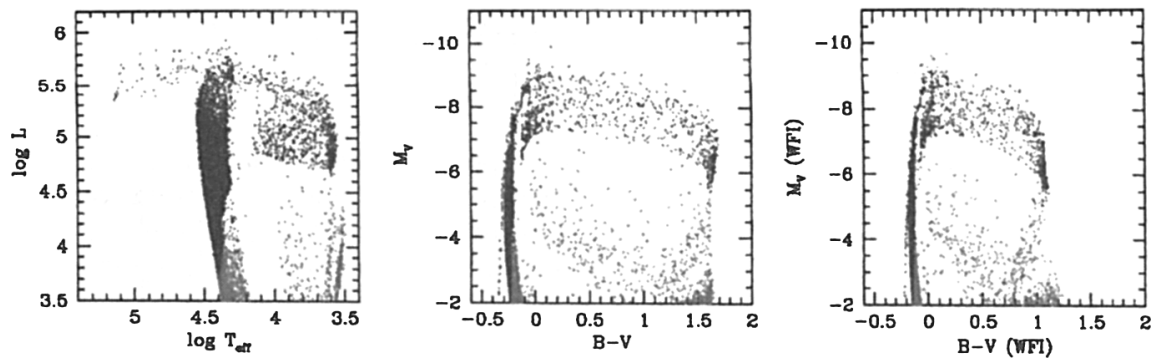

Figure 2. Synthetic CMDs for an intense and continuous burst of star formation between 4 and $15 \mathrm{Myr}$ (upper dots), overimposed to a 'typical' LMC field population (bottom dots). The H-R diagram is converted into CMDs in either the standard Johnson-Cousins-Glass system, or in the VEGA mag system of the ESO/MPI-WFI camera. Notice the appreciable changes in $B-V$ colours between these two systems, caused by the quite different filter transmission curves.

ism also allows us to consistently estimate extinction coefficients in the several passbands, as a function of stellar parameters and total visual extinction.

All results are being added to the web page http://pleiadi.pd.astro.it. It now includes extended tables of isochrones, bolometric corrections, and integrated colours of single-burst stellar populations.

\section{References}

Bertelli, G., Bressan, A., Chiosi, C., Fagotto, F., Nasi, E. 1994, A\&AS 106, 275

Girardi, L., Bressan, A., Bertelli, G., Chiosi, C. 2000, A\&AS 141, 371

Girardi, L., Bertelli, G., Bressan, A., Chiosi, C., Groenewegen, M.A.T., Marigo, P., Salasnich, B., Weiss, A. 2002, A\&A 391, 195

de Jager, C., Nieuwenhuijzen, H., van der Hucht, K.A. 1988, A\&AS 72, 259

Kudritzki, R.-P., Pauldrach, A., Puls, J., Abbott, D.C. 1989, A\&A 219, 205

Marigo, P., Girardi, L., Chiosi, C., Wood, P.R. 2001, A\&A 371, 152 